\title{
Characterization of double- deformable-mirror adaptive optics for IR beam shaping in hyperspectral imaging
}

Mohammad Azizian Kalkhoran, Ann Fitzpatrick, A. Douglas Winter, Chris Kelley, Edmund Warrick, et al.

Mohammad Azizian Kalkhoran, Ann Fitzpatrick, A. Douglas Winter, Chris S. Kelley, Edmund Warrick, Mark D. Frogley, Gianfelice Cinque, "Characterization of double-deformable-mirror adaptive optics for IR beam shaping in hyperspectral imaging," Proc. SPIE 11351, Unconventional Optical Imaging II, 113511A (4 May 2020); doi: 10.1117/12.2554162 


\title{
Characterization of double-deformable-mirror adaptive optics for IR beam shaping in hyperspectral imaging
}

\author{
Mohammad Azizian Kalkhoran ${ }^{\mathrm{a}}$, Ann Fitzpatrick ${ }^{\mathrm{a}}$, A.Douglas Winter ${ }^{\mathrm{a}}$, Chris S. Kelly ${ }^{\mathrm{a}}$, \\ Edmund Warrick ${ }^{\mathrm{a}}$, Mark D. Frogley ${ }^{\mathrm{a}}$, and Gianfelice Cinque ${ }^{\mathrm{a}}$ \\ ${ }^{a}$ Diamond Light Source, Harwell Science and Innovation Campus, Chilton, Oxfordshire, UK
}

\begin{abstract}
Vibrational microspectroscopy via Fourier transform infrared (FTIR) faces an experimental trade-off among the signal to noise ratio (SNR), acquisition time, spatial resolution, and sample coverage. This is mainly associated with broadband source type: e.g. low brightness thermal sources with high flux for large field of view imaging at low resolution, or low étendue of synchrotron radiation infrared (SRIR) for diffraction-limited scanning microanalysis at high magnification. ${ }^{1}$ Adaptive optics (AO), in this case deformable mirror (DM), is a potent tool in tackling the problem by modulating the intensity of high brightness structured SRIR beam toward a homogeneous field illumination for IR imaging at high magnification. The latter is required for an efficient coupling of SRIR source to a multi-pixel detector such as focal plane array (FPA). ${ }^{2}$ Additionally, DM enables to achieve different shapes, optimized for different Cassegrain IR objective. Regardless, the quality of the generated beam relies upon the performance of the adaptive elements, i.e. actuators and their linear and reproducible response to the applied voltage. Moreover, the beam shaping capability of a single DM in controlling light beam position and angle is limited by its actuators influence function. In this work, we implemented two DMs for intensity shaping for the complex SRIR beam. A variation of multi-conjugate AO is implemented to characterize the performance of DMs and their actuators transfer function at multiple locations. An IR sensitive microbolometer array has been optically conjugated to the focal plane of individual actuators and the far-field of DM, in order to probe the corresponding actuating response. By analysing each actuator's response individually, a measure of linear independence, uniformity in response, and cross-coupling can be obtained in a spectral range, from visible to near and mid IR. Additionally, by assembling the vectorized version of each actuator response, the transfer matrix can be formed. This matrix describes the relationship between the actuation effect on the beam and the response of the IR microbolometer, at the given conjugate planes. Based on such discussion, we assess the stability of the deformable mirror for open-loop (i.e. without feedback) operation.
\end{abstract}

Keywords: Synchrotron radiation Fourier transform infrared spectroscopy, deformable mirror, adaptive optics, focal plane array, hyperspectral imaging.

\section{INTRODUCTION}

Chemical imaging is an emerging class of methods which interrogate the sample microscale properties based on their molecular composition. Synchrotron Fourier transform infrared (SR-FTIR) microspectroscopy occupies a significant niche in this class that allows for high sensitive label-free measurements at very low concentrations. Micro-FTIR is a hybrid technique, which combines the merits of confocal microscopy spatial resolution with the infrared vibrational spectroscopy specificity. The latter is associated with the electric dipole moment of molecular bonds, mostly resonating in the band between mid-IR $2 \mu \mathrm{m}$ and $20 \mu \mathrm{m}$ and often referred to as the molecular fingerprint region. A broadband light source allows highly multiplexed spectral encoding, by means of an interferometer, to probe a wide range of vibrational molecular modes and overtone bands to classify the chemical composition and conformation of a wide range of materials in different disciplines such as biomedical diagnostics, ${ }^{3}$ gas-solid reactions, ${ }^{4}$ nanospectroscopy,${ }^{5}$ and metal-organic framework, ${ }^{6}$ to name but a few. Detection limits for far-field infrared microspectroscopy are governed by an interplay between the wavelengthdependent Abbe limit, étendue of the system and the sensitivity of the detector. Generally in the mid-IR,

Further author information: (Send correspondence to M.A.K)

M.A.K.: E-mail: mohammad.azizian@diamond.ac.uk

Unconventional Optical Imaging II, edited by Corinne Fournier, Marc P. Georges, Gabriel Popescu, Proc. of SPIE Vol. 11351, 113511A - (c) The Authors. Published under a Creative Commons Attribution CC-BY 3.0 License · doi: 10.1117/12.2554162 


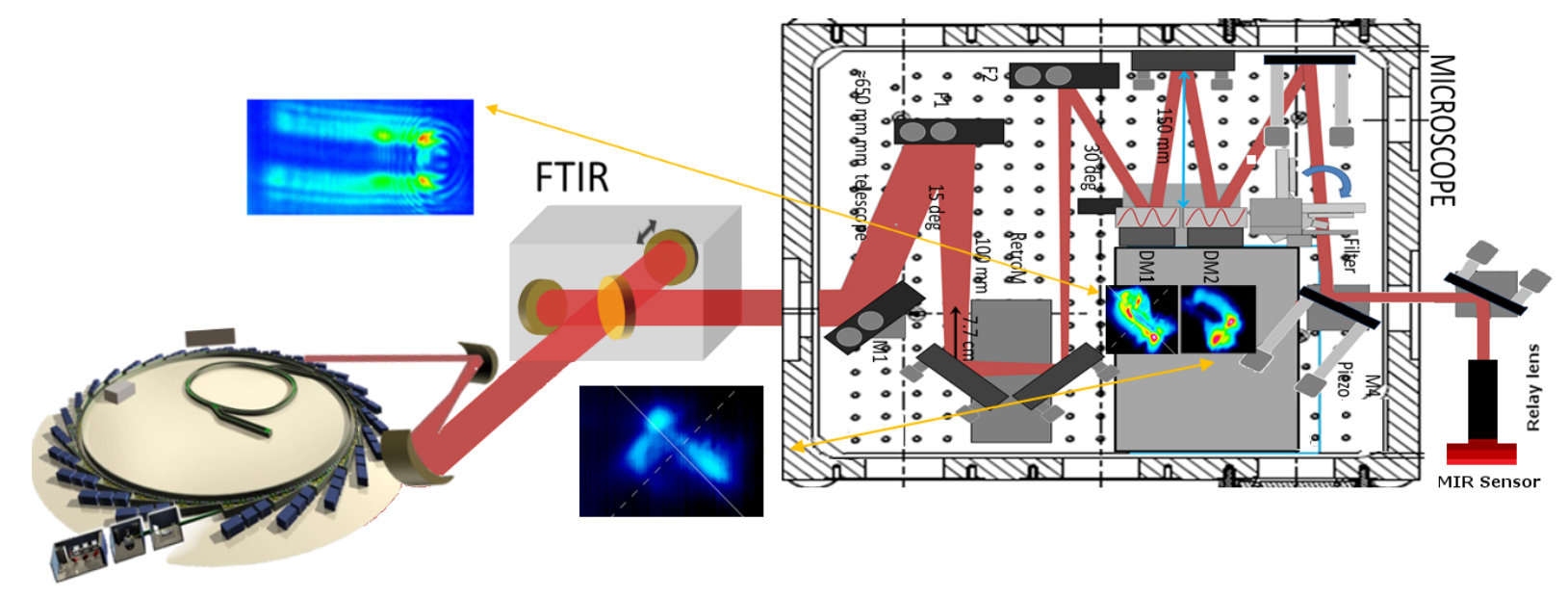

Figure 1: The schematic of adaptive optics vacuum box containing the conjugating telescope and deformable mirrors. DM1 and DM2 are conjugated to different plane, forming a multiconjugate adaptive optics. The monitoring system is placed outside of the vacuum box to monitor the performance of DMs remotely.

cryogenically cooled mercury cadmium telluride (MCT) single-element detectors are being used as state-of-theart high sensitive sensors. By mounting the sample on a motorized stage, molecular maps of scanned region can be reconstructed based on the collected spectra at the focal point. The multichannel recording capability of focal plane array (FPA) enables the extension of microspectroscopy to hyperspectral imaging and expedites the acquisition time, albeit at the expense of signal to noise ratio (SNR) and spatial resolution. ${ }^{7}$ Synchrotron IR (SRIR) radiation, with three order of magnitude higher brilliance than thermal source, one order of magnitude larger spectral distribution than laser sources ${ }^{8}$ and superior spectral stability to tunable lasers, is an ideal choice for FTIR microspectroscopy and imaging. In addition, the low étendue of SRIR benefits FTIR such that the diffraction-limited resolution with high spectral quality can be achieved for both single detector ${ }^{9}$ and FPA. To cope with the photon flux loss in confocal geometry, a high magnification objective (i.e.74x) is coupled to the FPA such that the oversampling can be implemented to realise the diffraction limited resolution with reasonable SNR level. ${ }^{2}$ The key-factor in full-field FPA hyperspectral imaging is the design of beamline optics to optimally match the extracted SR light from bending magnet to the pupil plane of the microscope Cassegrain objective. While this has previously demonstrated by splitting the large extraction angle and rescrambling the beamlets to fill the focal plane homogeneously, ${ }^{2,10}$ the approach is not feasible for the 3rd generation light sources such as DLS ${ }^{11,12}$ due to the smaller opening angle of dipole magnet radiation and inherently structured source of the SRIR, changing the form of the focal intensity distribution beyond its ideal diffraction-limited nature. For this reason we seek an alternative approach to gain more flexibility in beam shaping by integrating deformable mirrors (DMs) to modulate the intensity of the beam through control over the spatial intensity distribution and adaptive parallelisation of the rays to generate optimised beam shape quality for the given Cassegrain objective. In this work, we implemented two DMs in order to provide enough optical path length for correcting the intensity and angle for the entire mid-IR bandwidth. A variation of multi-conjugate AO is implemented to correct for SRIR structure and aberration by conjugating the DMs to corresponding planes. Our strategy falls in the realm of sensorless adaptive optics, where the need for wavefront sensor is circumvented by a control method, which relies on the performance of DM and its constituting elements, i.e. electromagnetic actuators controlling the shape of achromatic reflective membrane. The beam shaping capability of a single DM in controlling light beam intensity and angle is governed by its actuators influence function, the linearity and reproducibility of the actuator response to the applied voltage and the membrane stiffness whose propagate the deformation of the actuators beyond their neighbours. Even-though the microelectromechanical systems (MEMS) technology has overcome the observed hysteresis in piezo-electric actuators, the inter-actuator coupling caused by e.g. the physical mirror elastic material leads to the non-linear surface response and complicates the transfer function and modal response. ${ }^{13}$ 
In order to characterize the performance of the two DMs and their actuators transfer function, an IR sensitive microbolometer array (WincamD-IR-BB Dataray Inc.) has been optically conjugated to the focal plane of individual actuators and the far-field of the DMs. By analysing each actuator's response individually, a measure of linear independence, uniformity in response, and cross-coupling is obtained in different spectral range, from visible to near and mid IR. Additionally, by assembling the vectorized version of each actuator response, the transfer matrix (forward model) has been formed. This matrix describes the relationship between the actuation effect on the beam and the response of the IR microbolometer, at the given conjugated plane. By using the principal component analysis (PCA), the eigenmodes of the DMs can be reconstructed and utilised as a set of modal basis for either of model based reconstruction or guided closed loop algorithms. Based on these discussions, we assess the stability of the deformable mirror for open-loop (i.e. without feedback) operation.

\section{OPENLOOP CHARACTERISATION}

To characterise the fundamental responses of DMs, the light of an internal source of the FTIR system (Bruker Vertex $80 \mathrm{~V}$ ) is collimated and redirected to a vacuum box situated between FTIR and microscope (Hyperion 3000), where the adaptive optics instrumentation is installed (figure 1). The beam is downsized to match the DM pupil by a reflective telescope consisting of a pair of bare-gold coated parabolic mirrors and a retroreflector (2-flat mirrors). DMs are placed before the telescope such that each DM is conjugated to a separate plane behind the telescope. The characterized DMs are a pair of ALPAO DM97-15 having 97 actuators over a circular diameter of $13.5 \mathrm{~mm}$ with nominal pitch of $1.5 \mathrm{~mm}$ and a gold coated membrane. By working around a set of bias voltages, normalized to a scale from -1 to 1 , the magnetic actuators allow for bidirectional forces to be applied and generate a \pm 30 um max tilt across the DM surface. An adjustable two lens relay system has been designed to amplitude-conjugate the broadband $(2-16 \mu \mathrm{m})$ mid-IR sensor to the desired plane. For practicality, this monitoring system is placed outside of the vacuum box.

The optical relay system, composed of two zinc selenide aspheric lenses, is designed for remote monitoring of DM performance, in particular for the two following planes: a) near the DMs surface where the individual actuator focal spot forms b) at far-field plane where DM correction is desired, e.g. the surface of the second DM and the pupil of Cassegrain objective lens for first and second DM respectively. While the conjugation is confirmed by placing a clear optical path 1951 USAF microscope target, the confidence on the alignment is developed by applying an asterisk map to the corresponding DM with isotropic response of poked actuators (figure 2). Therefore, the monitoring system maps the change in the beam intensity distribution caused by the deformable mirror response to poked actuators to the sensor space. To determine the linearity of changes in the intensity caused by an actuator response to the applied bias, the response of a single actuator was measured over a range of applied bias from -1 to 1 , with an increment of 0.1 . A reference measurement with all actuators set to a reference bias of zero was subtracted from the recorded data. This way of measuring the influence function results in some compromise to its scalability which can be addressed by averaging the recorded intensity within a region corresponding to the map of the actuator over the conjugated plane. This process is repeated independently for every actuator in the deformable mirror. Figure 3 (video) depicts the measured far-field responses of a central actuator (figure 3a) as opposed to a marginal actuator (figure 3b). Aside from the substantial observed induced cross-coupling by the marginal transducers, the nonlinear response of the transducer makes it less valuable to be
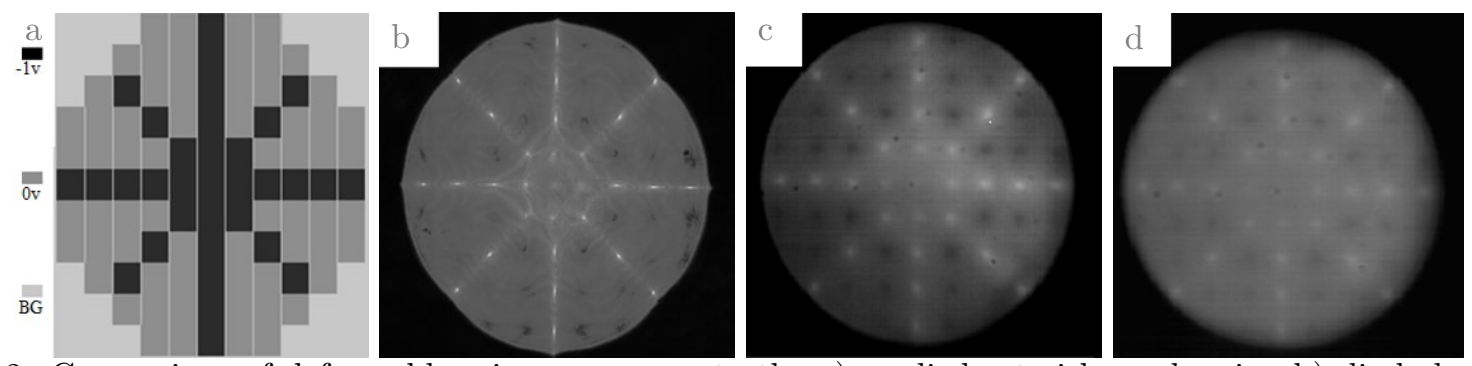

Figure 2: Comparison of deformable mirror response to the a) applied asterisk mask using b) diode laser (635 $\mathrm{nm}$ ) and a CCD camera c) near infrared thermal source and microbolometer array and d) mid-infrared thermal source and microbolometer array. 
considered for e.g. closed-loop optimisation algorithm. Of note, for driving bases using PCA requires the closed under multiplication criterion (i.e. linear scalability) to hold which means that the accuracy of the influence function for least-squares fitting depends on its ability to scale accurately. Figure 4a shows the linearity of all actuators in matrix representation, where the most linear behaviour are mainly associated with the central actuators while marginal actuators and those in close proximity are behaving differently. Figure $4 \mathrm{~b}$ depicts the uniformity of the actuators across the DM for the applied -1 bias voltage, by deforming the first row of figure $4 \mathrm{a}$ to the shape of deformable mirror.
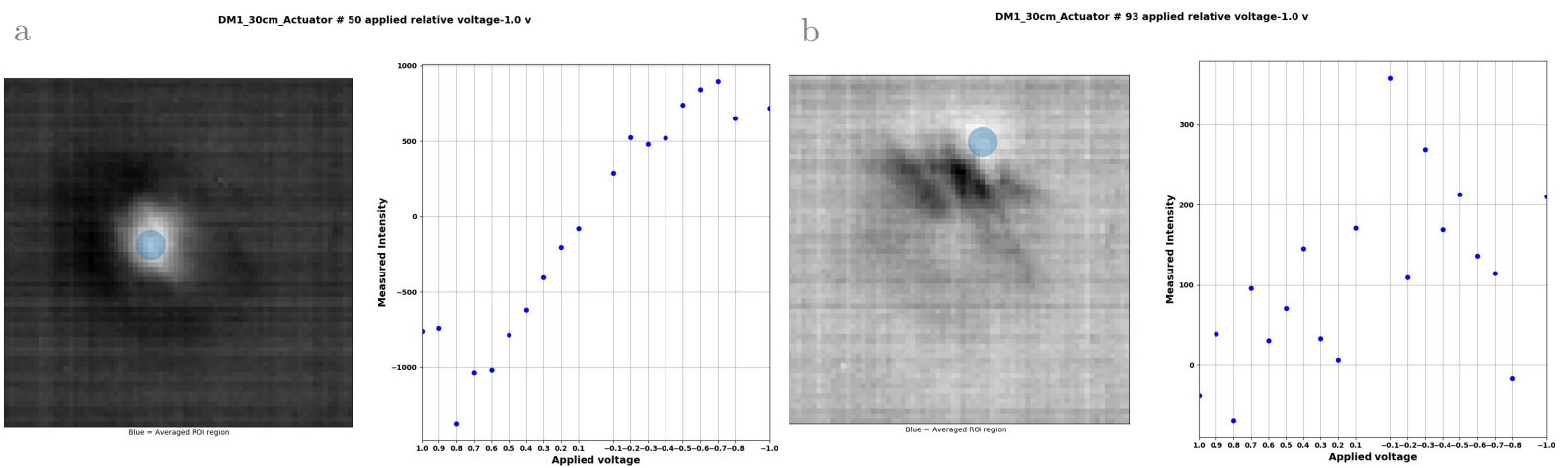

Figure 3: Video 1. Visualisation of spatial variant response of the deformable mirror to the applied bias voltage for a) central actuator and b) marginal actuator. The overlaid blue circle represents the averaged region considered for each measurement on the graph:

http://dx.doi.org/10.1117/12.2554162.1

http://dx.doi.org/10.1117/12.2554162.2

Another approach to assess the DM performance includes interaction between influence functions of nearby actuators, an indication for cross-coupling effect, which may restrict the linear independence range required for constructing the modal basis. The application of a force by an actuator on the membrane results in a finite displacement at the positions of neighboring actuators as well. From figure 2, the cross-coupling effect is evident and that is regardless of the nature of the beam. To measure the complete shape of produced distortion of the beam by a single actuator, the imaging plane is conjugated to the surface of the DM and the level of induced distortion has been measured (figure 5a). To study the actuator response additivity, several random pairs of neighbouring actuators across the deformable mirror are tested. Here, the results for the pair of $48^{t h}$ and $49^{t h}$ in push pull configuration is shown, which are representative of the other actuators. The question here is whether the distortion level is large enough to cause a different response from tandem poking of actuators than the summation of individual poking events. Figure $5 \mathrm{~b}$ demonstrate that the difference is negligible, regardless of the distance between the actuators. Therefore it is logical to assume that the deformation of the DM surface result from poking all actuators is the linear superposition of individual contribution from each actuator.
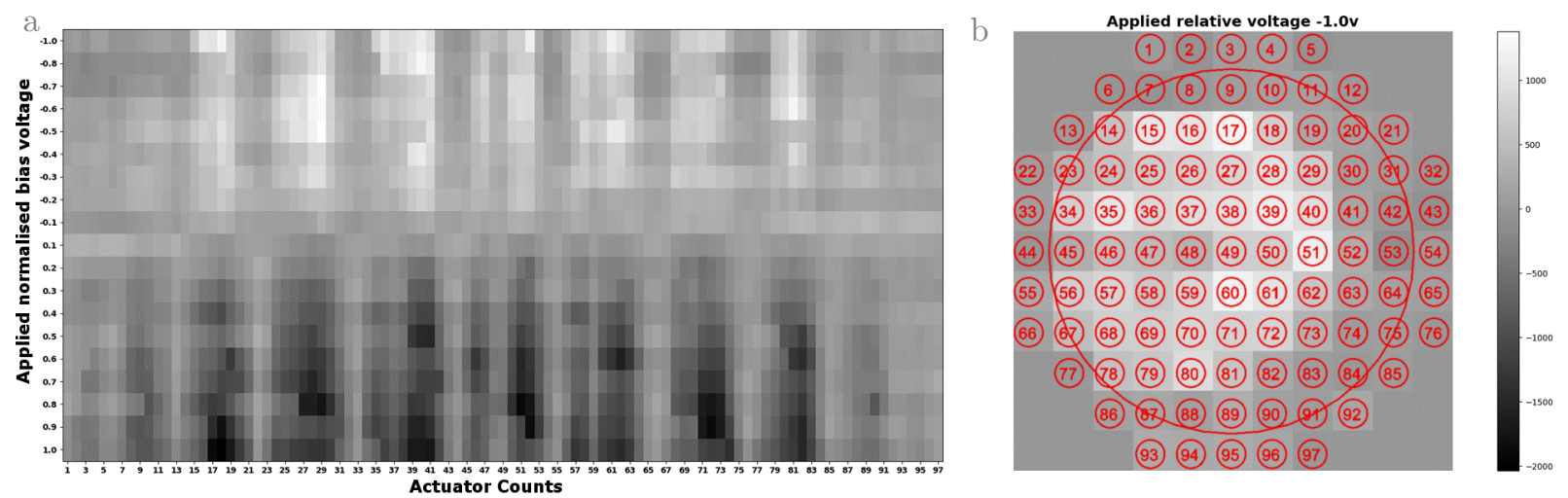

Figure 4: The deformable mirror actuators a) linearity and b) uniformity with respect to the applied bias voltages.

The effective surface of the deformable mirror is shown by the red circle. 


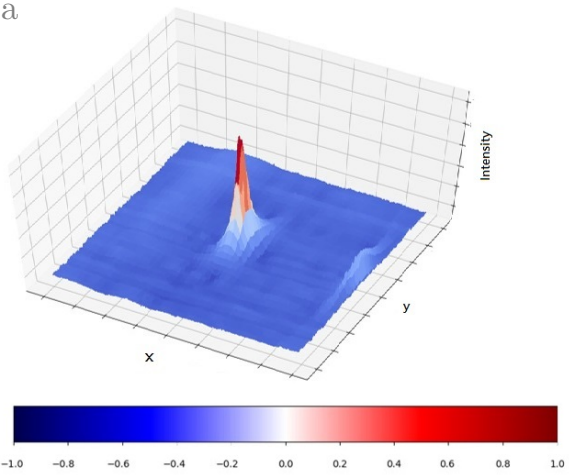

b

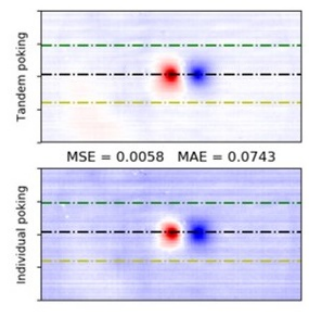

Central Actuator -1.0
1 Actuators apart 0.9
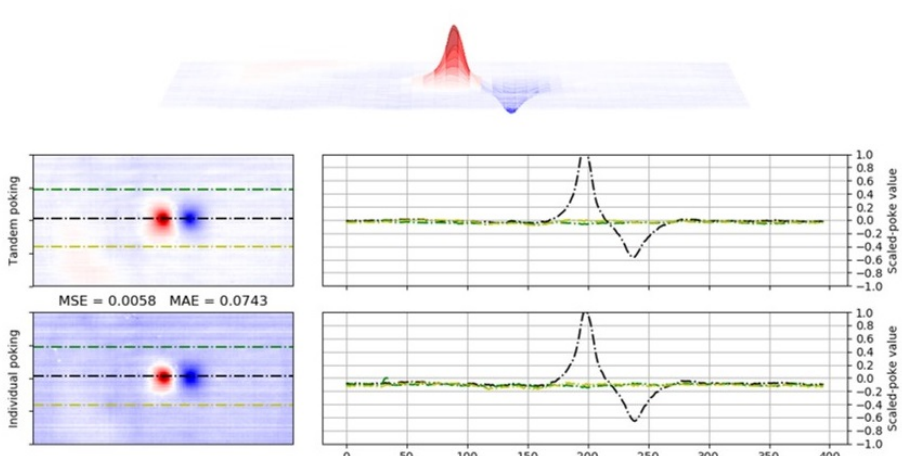

Figure 5: Visualisation of the inter-actuator coupling a) poking the central actuator by -1 bias voltage induces negligible strain on the neighbouring actuators, b) comparison between poking two adjacent actuators in tandem and individually.

An empirical transfer matrix can be generated in the form of a Casorati matrix whose columns are composed of vectorised recorded response of individual actuators. While each column of this matrix represent the recorded response to an actuator movement, its rows correspond to a single pixel of the sensor. This matrix describes the relation between measured responses of actuator and coefficient associated with the basis employed for least square, to replicate ideal DM shape. ${ }^{14,15}$ The column vectors of the transfer matrix, which represent the slope of the phase generated by the DM, form the natural (zonal) bases for DM beam shaping by iterative algorithms. However, due to the linear dependence between the columns of the matrix due to the cross-coupling effect, it is suboptimal choice for expressing the measured wavefronts. As the bases of this matrix are not orthogonal, we put forward the use of the eigen-decomposition of the transfer matrix operator to expand the actuators response into its eigenmodes in order to derive the modal bases which allows for the transfer matrix to be employed in least square estimation or to guide the iterative closed-loop optimization algorithms. The eigenmodes are mutually orthogonal bases with the same counts as the actuator number. Moreover, their associated singular values rank them based on their contribution (or their gain) to the shape of DM, hence the degree of freedom for searching algorithm can be limited to the most important modes. This was accomplished by using singular value decomposition of matrix M, given by $M_{m \times n}=U_{m \times m} \Sigma_{m \times n} V_{n \times n}^{T}$. Matrices $U$ and $V$ are orthonormal while columns of $U$ contains $n$ (same as actuator counts) columns depicting the eigenmodes of DM, $\Sigma$ is a diagonal matrix containing singular values $\sigma_{i}$ along its diagonal, representing the gain and order of modal bases and $V$ is a unitary matrix that permits the conversion between zonal and modal modes. In principle, the most salient features of the DM are stored in the first few bases $\left(M_{\text {prime }}\right)$ provided by the singular value spectrum where $\sigma_{i}>0$. Figure6 is the visualisation of the reconstructed eigenmodes. Theoretically, these eigenmodes can serve as bases for the optimisation algorithm, where searching can be concentrated on the low modes with higher gains and ignoring higher modes.
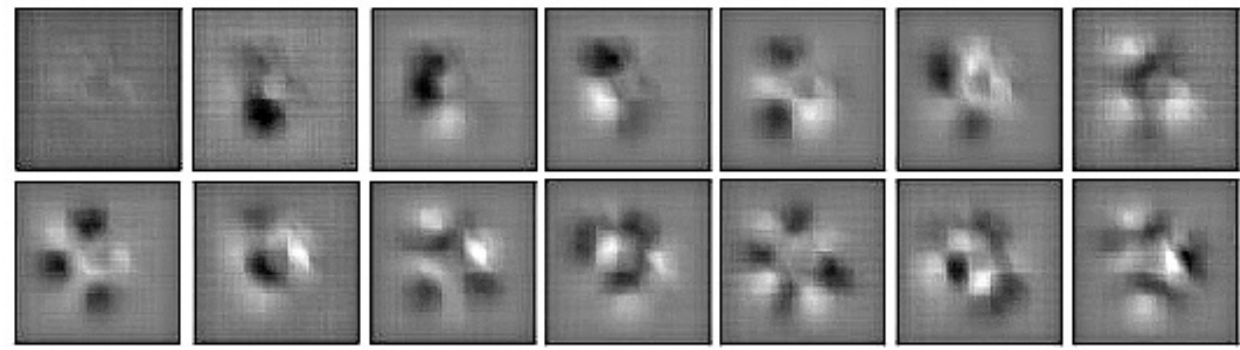

Figure 6: Visualisation of deformable mirror reconstructed far-field eigenmodes. Only 14 modes with largest singular values are shown. 


\section{CONCLUSION}

The focus of this work was design and characterisation of a high compelling adaptive optics for SRIR beam shaping. An underpinning step of this work was constructing the transfer matrix that explains the performance of the designed system. The calculation is based on the DM response to the actuator forces that relates the mirror space to the sensors. The purpose of developing the transfer matrix was mainly to characterise and further optimise the correction aptitude for the SRIR beam aberration and structure. While the measured data allowed to study the actuators response and derive a measure of linear independence, uniformity in response, and inter actuator cross-coupling, the effect if internal source on spatial variant response has not been taken into account. In terms of open loop characterisation, the derivation of eigenmode delivers a succinct representation of mirror deformations whilst satisfying the orthogonality condition for the given sensorless adaptive optics scheme, where analytic modes like Zernike or Lukosz modes are not ideal. These bases offer a more realistic model for controlling the DMs in both model based adaptive optics and closed loop algorithms.

\section{ACKNOWLEDGEMENT}

This work was funded by Diamond Light Source through in house beamtime at B22.

\section{REFERENCES}

[1] Baker, M. J. et al. Using fourier transform IR spectroscopy to analyze biological materials. Nature protocols 9, 1771 (2014).

[2] Nasse, M. J. et al. High-resolution fourier-transform infrared chemical imaging with multiple synchrotron beams. Nature methods 8, 413 (2011).

[3] Movasaghi, Z., Rehman, S. \& ur Rehman, D. I. Fourier transform infrared (FTIR) spectroscopy of biological tissues. Applied Spectroscopy Reviews 43, 134-179 (2008).

[4] Schliesser, A., Picqué, N. \& Hänsch, T. W. Mid-infrared frequency combs. Nature Photonics 6, 440 (2012).

[5] Donaldson, P. M. et al. Broadband near-field infrared spectromicroscopy using photothermal probes and synchrotron radiation. Optics express 24, 1852-1864 (2016).

[6] Smith, G. L. et al. Reversible coordinative binding and separation of sulfur dioxide in a robust metal-organic framework with open copper sites. Nature materials 18, 1358-1365 (2019).

[7] Della Ventura, G. et al. FTIR imaging in diffusion studies: Co2 and h2o in a synthetic sector-zoned beryl. Frontiers in Earth Science 3, 33 (2015).

[8] Bechtel, H. A., Muller, E. A., Olmon, R. L., Martin, M. C. \& Raschke, M. B. Ultrabroadband infrared nanospectroscopic imaging. Proceedings of the National Academy of Sciences 111, 7191-7196 (2014).

[9] Chan, K. A., Fale, P. L., Atharawi, A., Wehbe, K. \& Cinque, G. Subcellular mapping of living cells via synchrotron microFTIR and ZnS hemispheres. Analytical and bioanalytical chemistry 410, 6477-6487 (2018).

[10] Stavitski, E. et al. Dynamic full-field infrared imaging with multiple synchrotron beams. Analytical chemistry 85, 3599-3605 (2013).

[11] Tobin, M. et al. Focal plane array IR imaging at the australian synchrotron. Infrared Physics \& Technology 94, 85-90 (2018).

[12] Quaroni, L., Zlateva, T., Wehbe, K. \& Cinque, G. Infrared imaging of small molecules in living cells: from in vitro metabolic analysis to cytopathology. Faraday discussions 187, 259-271 (2016).

[13] Bitenc, U., Bharmal, N. A., Morris, T. J. \& Myers, R. M. Assessing the stability of an alpao deformable mirror for feed-forward operation. Optics express 22, 12438-12451 (2014).

[14] Shaw, M., Hall, S., Knox, S., Stevens, R. \& Paterson, C. Characterization of deformable mirrors for spherical aberration correction in optical sectioning microscopy. Optics express 18, 6900-6913 (2010).

[15] Wang, B. \& Booth, M. J. Optimum deformable mirror modes for sensorless adaptive optics. Optics Communications 282, 4467-4474 (2009). 